biomarkers to classify disease phenotypes relevant to the onset of diabetic steatosis followed by progression towards liver inflammation.

Methods We compared miR-192-3p level in mice given HFD (high-fat diet), HFFD (high-fat fructose diet), and MCD (methionine-choline deficient diet) diets. Histopathological lesions were analyzed at critical stages to correlate the miRNA expression. In obese individuals diagnosed with DM or not, we examined the miR-192-3p level by droplet digital PCR and characterized serum whole transcriptomes with SEQuoia Complete Stranded RNA Library kits.

Results Along with our reported loss of the overlooked star strand miR-192-3p in $\mathrm{db} / \mathrm{db}$ mice, we found a high circulating level of miR-192-3p in different diets-induced animals, indicating a heightened inflammatory response in the liver, especially in those given HFFD and MCD diets. Since circulating miR192-3p surges only in HFFD under an insulin-resistant state but not manifested at all in HFD, its circulating level shall reflect the extent of runaway glycerolipid metabolism. Indeed, we observed a similar expression pattern in serum miR-192$3 p$ for obese individuals $(\mathrm{BMI}>34)$, where the miRNA can only be detected in individuals diagnosed with DM. Our transcriptomic analysis identified that PI3K-Akt signaling, FoxO signaling and TGF $\beta$ signaling pathways were enriched in the hepatocytes with miR-192-3p overexpression. The top differential circulating non-coding transcripts between those individuals with or without diabetes belonged to the ribosomal pathway under the modulating actions of Akt, suggesting the use of these transcripts for a better account of diabetic phenotype.

Conclusions Circulating levels of miR-192-3p and transcripts along its regulated Akt pathway account for visceral obesity and reflect prognostic outcomes to the progression of diabetes. We hereby advocate miR-192-3p as a functional glycerolipid regulator for steatosis in the diabetic liver which offers know-how for better disease staging and treatment regimes.

\section{IDDF2021-ABS-0204 SARS-COV-2 PRODUCTIVELY INFECTS HUMAN LIVER AND BILIARY ORGANOIDS}

Yi Zhao*, Xiaoxue Ren, Xiaoxing Li, Lixia Xu, Jing Lu, Jun Yu. Institute of Precision Medicine, The First Affiliated Hospital, Sun Yat-sen University

\subsection{6/gutjnl-2021-IDDF.19}

Background SARS-CoV-2 has caused the COVID-19 pandemic, 14-53\% with COVID-19 developed hepatic dysfunction. More data is required to ascertain the pattern of liver injury in patients with COVID-19. Here we report the use of human liver and biliary organoids as a tool to investigate the SARS$\mathrm{CoV}-2$ infection and virus-induced tissue damage ex vivo at the cellular and molecular levels.

Methods Immunofluorescence staining and RT-qPCR was performed to examine the susceptibility and replication of human liver and biliary organoids to SARS-CoV-2. Transmission electron microscopy was performed to investigate the ultrastructure of SARS-CoV-2 infected biliary organoid. The mRNA sequence analysis was performed to determine gene expression changes induced by SARS-CoV-2-infection of biliary organoids.
Results Immunofluorescence staining showed that SARS-CoV-2 spike (S) glycoprotein protein was readily detected in patchy areas of the human liver and biliary organoids, whereas no signal was found in uninfected control. RT-qPCR showed that the SARS-CoV-2 genomic RNAs revealed a dramatic increase of viral load in organoids at $24 \mathrm{~h}$ post-infection $(\mathrm{MOI}=1)$ and it can remain stable 96 hours in the liver organoids and 48 hours in biliary organoids. Unsupervised transmission electron microscopy (TEM) showed that viral particles occurred in the lumen of the organoid, at the basolateral and apical side of the organoid, even in membrane-bound vesicles. Additionally, RNA-seq revealed early cell response to virus infection, including the well-known upregulation of chemokines and inflammatory cytokines.

Conclusions Therefore, the hepatocytes and bile duct cells have effectively been infected by COVID-19, and human liver and biliary organoids can serve as a pathophysiological model to investigate the underlying mechanism of SARS-CoV-2 infection.

\section{IDDF2021-ABS-0207 TYPE 2 RESISTANT STARCH IMPROVES LIVER STEATOSIS INDUCED BY HIGH-FAT DIET RELATING TO GUT MICROBIOTA REGULATION AND CONCENTRATION OF PROPIONIC ACID IN PORTAL VEIN BLOOD IN C57BL/6J MICE}

Diwen Shou*, Chuangyu Cao, Haoming Xu, Hongli Huang, Yu Xia, Qing Mei, Ying Quan, Hanqing Chen, Chong Zhao, Wenjuan Tang, Huiting Chen, Yongjian Zhou. Department of Gastroenterology and Hepatology, Guangzhou Digestive Disease Center, Guangzhou First People's Hospital, School of Medicine, South China University of Technology, Guangzhou, China

\subsection{6/gutjnl-2021-IDDF.20}

Background Resistant starch (RS) was approved to regulate glucose and lipid metabolism and gut microbiota in vivo. Our aim was to explore the effect and mechanism of type 2 resistant starch (RS2) on NAFLD mice.

Methods Twenty C57BL/6J male mice were randomly divided into $\mathrm{CD}$ group, CD+RS group, HFD group, HFD+RS group $(n=5)$. The NAFLD mouse model was induced by a high-fat diet for 16 weeks. RS2 was supplemented into the diet for 4 weeks subsequently. After treatment, liver steatosis was evaluated by HE staining and NAS score. The serum alanine aminotransferase (ALT), triglyceride (TG), fasting serum glucose (FBG) and insulin resistance index (HOMA-IR) of portal vein blood were detected. The intestinal content of mice was collected for microbiota analysis by $16 \mathrm{~s}$ amplicon sequencing. The level of short-chain fatty acids (SCFA) in portal vein blood was detected.

Results Compared with HFD group, after RS intervention, the liver steatosis of mice was significantly reduced, and the serum levels of ALT, TG, FBG and HOMA-IR were significantly decreased $(P<0.05)$ (IDDF2021-ABS-0207 Figure 1a Type 2 resistant starch improves liver steatosis induced by high-fat diet; Hepatic HE staining of mice) (IDDF2021ABS-0207 Figure 1b Type 2 resistant starch improves liver steatosis induced by high-fat diet; NAS score of mice $(* P<0.01))$ (IDDF2021-ABS-0207 Figure 1c Type 2 resistant starch improves liver steatosis induced by high-fat diet; Serum levels of ALT in mice $(* * P<0.01)$ (IDDF2021-ABS- 

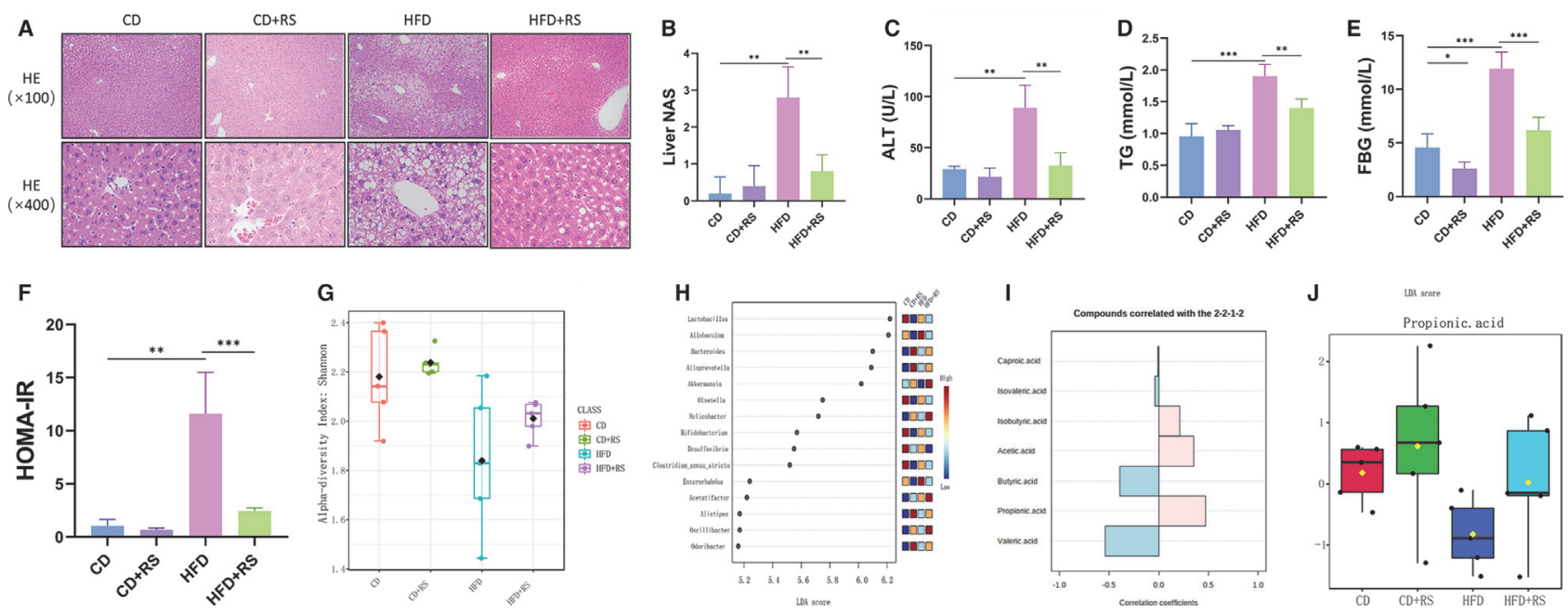

Abstract IDDF2021-ABS-0207 Figure 1

0207 Figure 1d Type 2 resistant starch improves liver steatosis induced by high-fat diet; Serum levels of TG in mice $(* P<0.01, * * P<0.001) \quad$ (IDDF2021-ABS-0207 Figure 1e Type 2 resistant starch improves liver steatosis induced by high-fat diet; Serum levels of FBG in mice $(" P<0.05$, $* * P<0.001))($ IDDF2021-ABS-0207 Figure 1f Type 2 resistant starch improves liver steatosis induced by high-fat diet; Serum levels of HOMA-IR in mice $(* * P<0.01$, $* * P<0.001)$ ). The $\alpha$ diversity of intestinal microflora was decreased in HFD group mice compared with CD group mice, but slightly increased after RS2 intervention (IDDF2021-ABS-0207 Figure 1g Type 2 resistant starch improves liver steatosis induced by high-fat diet; Alpha diversity). LEfSe analysis showed that the abundance of Akkermansia (LDA=6.02) was decreased in the HFD group mice compared with CD group mice and increased significantly after RS2 intervention, indicating that it was a biomarker of NAFLD mice (IDDF2021-ABS-0207 Figure 1h Type 2 resistant starch improves liver steatosis induced by high-fat diet; LEfSe analysis). The concentration of valeric acid in portal vein blood was negatively correlated with the abundance of Akkermansia $(r=-0.54, P=0.014)$, and propionic acid was positively correlated with the abundance of Akkermansia ( $\mathrm{r}=0.47, P=0.036)$ (IDDF2021-ABS-0207 Figure $1 \mathrm{i}$ Type 2 resistant starch improves liver steatosis induced by high-fat diet; Correlation analysis of SCFA and intervention) (IDDF2021-ABS-0207 Figure 1j Type 2 resistant starch improves liver steatosis induced by high-fat diet; Concentration of propanoic acid in portal vein blood. CD, control diet; HFD, high-fat diet; RS, type 2 resistant starch).

Conclusions RS2 intervention can improve liver steatosis, liver function, serum lipid and serum glucose levels and insulin resistance in NAFLD mice, The $\alpha$ diversity of intestinal microflora in RS2 treated mice was increased too. Akkermansia is a biomarker of NAFLD mice, which is significantly increased after RS2 treatment. The concentration of propionic acid in portal vein blood of mice increased after RS2 treatment and was positively correlated with the abundance of Akkermansia.

Diwen Shou and Chuangyu Cao contributed equally to this work.

\section{Basic Gastronenterology}

\section{IDDF2021-ABS-0013 THE METHOD FOR OBTAINING A REPRODUCIBLE MODEL OF TUBERCULOUS PERITONITIS IN RABBITS}

${ }^{1}$ Dmitry Plotkin*, ${ }^{2}$ Tatiana Vinogradova, ${ }^{3}$ Mikhail Reshetnikov, ${ }^{3}$ Mikhail Sinitsyn, ${ }^{4}$ Sergey Okovityi. ${ }^{1}$ Moscow Research and Clinical Center for TB Control, Pirogov Russian National Research Medical University of the Ministry of Healthcare of the Russian Federation, Moscow, Russia; ${ }^{2}$ Saint-Petersburg State Research Institute of Phthisiopulmonology of the Ministry of Healthcare of the Russian Federation, SaintPetersburg, Russia; ${ }^{3}$ Moscow Research and Clinical Center for TB Control Healthcare Department, Moscow, Russia; ${ }^{4}$ Saint- Petersburg State Chemical Pharmaceutical University of the Ministry of Healthcare of the Russian Federation, Saint-Petersburg, Russia

\subsection{6/gutjnl-2021-IDDF.21}

Background The unsatisfactory results of the diagnosis and treatment of tuberculosis peritonitis as well as the high rate of disability and mortality in this disease require further study of the pathogenesis of its progression, the features of morphology, and the development of new treatment methods. To this end, we have created a reproducible model of chronic tuberculous peritonitis to study the pathophysiological mechanisms of its development.

Methods Approved by the Local Ethics Committee, the study was performed using 10 male rabbits of the 'Soviet Chinchilla' breed. All the work with model animals was carried out in accordance with international standards for the humane treatment of laboratory animals. Rabbits underwent intraperitoneal administration of mycobacterium tuberculosis (MBT), modeling tuberculosis peritonitis according to our proposed method which included intravenous administration of TNF inhibitor and intraperitoneal inoculation of iron (III) hydroxide of the sucrose complex. As a result, local and general immunosuppression led to active development of MBT in the peritoneum layers causing specific inflammation. The animals were removed from the experiment on the 44th day after infection. Samples of the parietal and visceral peritoneum, intestines, lungs, lymph nodes, parenchymal organs were collected for morphological examination. 